\title{
Les déterminants de la confiance/méfiance du consommateur marocain vis-à-vis du cyber-achat
}

\author{
Abir Bercheq, (Docteur en sciences économiques)
}

Laboratoire de Recherche sur l'entreprenariat et management de l'environnement des entreprises

Faculté des sciences Juridiques, Economiques et Sociales de Mohammedia

Université Hassan II de Casablanca, Maroc

\section{Samira Oukarfi, (Professeur de l'enseignement supérieur)}

Laboratoire de Recherche en Management, Information et Gouvernance

Faculté des sciences Juridiques, Economiques et Sociales Aïn Sebaa

Université Hassan II de Casablanca, Maroc

Youssef Jamal, (Professeur de l'enseignement supérieur)

Laboratoire de Recherche sur l'entreprenariat et management de l'environnement des entreprises

Faculté des sciences Juridiques, Economiques et Sociales de Mohammedia

Université Hassan II de Casablanca, Maroc

Doi:10.19044/esj.2020.v16n4p131～URL:http://dx.doi.org/10.19044/esj.2020.v16n4p131

\section{Résumé}

Cet article propose une étude empirique des déterminants de la confiance vs. méfiance des consommateurs marocains à l'égard du cyberachat. Ce secteur peine à se développer au Maroc en raison de plusieurs facteurs, notamment l'absence de compétences numériques, la fiabilité des vendeurs en ligne et la faiblesse d'un cadre législatif protégeant les intérêts des consommateurs en ligne. C'est dans ce cadre que se situe ce papier. Un questionnaire a été administré auprès de 2254 individus localisés dans trois villes marocaines différentes. Une analyse factorielle multidimensionnelle a relevé l'existence d'une méfiance envers l'achat en ligne chez les marocains due à plusieurs facteurs. De plus, cette étude a permis de classer les individus en trois groupes selon les variables les plus discriminantes : "Les cyberconsommateurs confiants» qui achètent sans réserve, les «cyberconsommateurs méfiants » qui achètent en ligne mais qui pensent être conscients du danger que représente l'achat virtuel et les « nonconsommateurs » présentant une aversion au commerce électronique.

Mots clés : Commerce en ligne, confiance en ligne, méfiance, risque perçu, analyse factorielle multidimensionnelle 


\title{
Determinants of the Trust/Mistrust of Moroccan Consumer Towards E-Commerce
}

\author{
Abir Bercheq, (Docteur en sciences économiques)
}

Laboratoire de Recherche sur l'entreprenariat et management de l'environnement des entreprises

Faculté des sciences Juridiques, Economiques et Sociales de Mohammedia

Université Hassan II de Casablanca, Maroc

\section{Samira Oukarfi, (Professeur de l'enseignement supérieur)}

Laboratoire de Recherche en Management, Information et Gouvernance

Faculté des sciences Juridiques, Economiques et Sociales Aïn Sebaa

Université Hassan II de Casablanca, Maroc

\section{Youssef Jamal, (Professeur de l'enseignement supérieur)}

Laboratoire de Recherche sur l'entreprenariat et management de l'environnement des entreprises

Faculté des sciences Juridiques, Economiques et Sociales de Mohammedia

Université Hassan II de Casablanca, Maroc

\begin{abstract}
This article proposes an empirical study of the determinants of trust vs. distrust of Moroccan consumers towards e-commerce. This sector is struggling to develop in Morocco due to several factors, including the lack of digital skills, the reliability of online sellers and a weakness of legislative framework protecting the interests of online consumers. It is against this background that our contribution is situated. We opted for an empirical approach by administering a survey to 2254 individuals located in three different Moroccan cities. A multidimensional factorial analysis revealed the existence of a mistrust towards e-commerce among Moroccans due to several factors. In addition, this study allowed us to classify individuals into three groups according to the most discriminating variables: "Confident cyber-consumers", "mistrustful cyber-consumers" and "online non-shoppers" with an aversion to e-commerce.
\end{abstract}

Keywords: E-commerce; E-trust; Mistrust; perceived risk; multi-component analysis 


\section{Introduction}

Depuis plusieurs décennies, le monde connaît une véritable révolution numérique qui a profondément bouleversé les modes de production et de consommation. L'avènement de la technologie internet a refaçonné les styles de vie des sociétés. La mise en réseau planétaire des individus et des organisations s'est traduite par l'abolition de la distance physique pour accéder à de nouveaux produits et services proposés en ligne. Le commerce électronique est désormais considéré comme un substitut à l'achat classique en raison des multiples avantages qu'il procure à ses adeptes. A cet égard, le rapport de la Conférence des Nations Unies sur le Commerce et le Développement (CNUCED) avance que plus de $70 \%$ de la population des pays développés achète des biens et services en ligne alors que dans les pays les moins avancés elle reste inférieure à $2 \%$.

Le Maroc, malgré le fait qu'il occupe une honorable cinquième place en matière de commerce électronique au niveau du continent africain, il reste à la traîne en étant classé par la CNUCED à la $81^{\text {ème }}$ place sur les 151 pays évalués sur la base de l'indice du commerce électronique «Business to Consumer » (B2C). Beaucoup de progrès restent à faire afin de stimuler le secteur et inciter le consommateur marocain à adopter ce nouveau mode de consommation. En effet, la réticence à l'égard du cyber-achat au Maroc, comme c'est le cas dans plusieurs pays en développement, peut s'expliquer par plusieurs facteurs, entre autres, l'absence d'aptitudes et de compétences numériques et la faiblesse d'un cadre législatif et réglementaire protégeant les intérêts des protagonistes de l'échange en ligne. L'apparition et la multiplication des risques sur la sécurité des données personnelles ont créé un sentiment de méfiance à l'égard de tout de ce qui relève du numérique. Instaurer la confiance en ligne est désormais devenue la préoccupation majeure des entreprises qui opèrent sur la toile.

Ce fait stylisé a suscité un foisonnement de travaux théoriques et empiriques sur la question de la confiance en l'achat en ligne. La majorité des travaux s'accordent à dire que la confiance est le principal déterminant de l'adoption du commerce électronique (Hine et Eve, 1998 ; Lee et Turban, 2001 ; McKnight et Chervany, 2001 ; Belanger et al., 2002 ; McKnight et al., 2002 ; Pavlou, 2003). La nature virtuelle de l'achat en ligne, l'absence de contact humain, le risque de sécurité des données privées, accompagnés de l'intangibilité des produits à acheter font de la confiance un antécédent du commerce électronique (Gefen, 2000 ; Jarvenpaa et al., 2000 ; McKnight et al., 2002 ; Pavlou, 2003 ; Chouk, L. et Perrien, 2005)

C'est dans ce cadre que se situe cette contribution qui s'attèle à expliquer les origines de la confiance/méfiance des consommateurs marocains à l'égard du commerce électronique. Ce papier tente de répondre aux interrogations suivantes : 
- Existe-t-il une cyber-méfiance à l'encontre de l'E-achat au Maroc ? serait-elle à l'origine de la non-acceptation du e-commerce ?

- La méfiance serait-elle liée à une asymétrie d'information entre acheteurs et vendeurs en ligne ?

- Les conditions socioéconomiques des individus auraient-elle un impact sur la confiance en l'achat en ligne?

Ce travail sera structuré comme suit. La première section sera consacrée à la revue de la littérature sur les déterminants de la confiance dans le contexte de l'achat en ligne. La confiance est un concept multidimensionnel dont les déterminants vont des caractéristiques du site web et du marchand en ligne jusqu'à ceux du consommateur qui jouent un rôle majeur. La notion de confiance en ligne amène aussi à l'étude du concept de risque. Le risque perçu et l'incertitude représentent des défis majeurs dans l'intention du consommateur à placer sa confiance dans l'acte d'achat sur internet. La deuxième section présente l'enquête et les premiers résultats à plat. La troisième section est consacrée aux résultats de l'analyse en composantes multiples.

\section{Les Déterminants Théoriques Et Empiriques De La Confiance Numérique}

\subsection{Eléments De Définition}

La confiance est concept complexe qui revêt plusieurs facettes. Sa conceptualisation dépend de son contexte et des approches utilisées (Lewis et Weigert, 1985 ; McAllister, 1995 ; Guibert, 1999 ; Nissenbaum, 2001 ; Singh Sabol, 2002). Certains auteurs considèrent la confiance comme l'intention qui précède le comportement (Rousseau et al., 1998 ; McKnight et al., 2002), d'autres comme une émotion entrant dans les relations interpersonnelles (Mayer et al., 1995) et certains pensent que la confiance est une action et une prise de décision dans un contexte risqué (Deutsch, 1962 ; McAllister, 1995).

La confiance numérique se distingue de la confiance traditionnelle par le contexte virtuel très spécifique dans lequel se conclue la transaction. La particularité réside dans l'incertitude dans laquelle sont menés les échanges. Marouseau (2003) énumère cinq points de différence entre la confiance en l'achat ligne et la confiance traditionnelle : «l'absence de vendeurs, l'absence d'interactions avec les autres clients, la nécessité de s'adapter à la structure de pensée de l'internaute, l'absence d'informations poly-sensorielles pour évaluer le produit et coût de la recherche d'information ». En outre, les transactions en ligne sont impersonnelles et automatisées (Head et Hassanein, 2002). Cette absence d'interactions humaines dans les échanges commerciaux est accompagnée d'incertitude, de possibilité de fraude et d'abus. L'appréhension des consommateurs est accentuée par les problématiques de 
protection de données personnelles et de la vie privée des internautes (Hine et Eve, 1998).

Le concept de « risque » est omniprésent dans les attributs de la confiance en ligne puisqu'il joue un rôle majeur entre la phase de l'information et la phase de l'achat en ligne (Novak et Peralta, 2000). Chouk et Perrien (2003) distinguent entre deux types de risques : le risque comportemental et le risque environnemental. Le risque comportemental est principalement lié au potentiel comportement opportuniste du marchand dû à une asymétrie d'information (fausses déclarations, publicités mensongères). Le risque environnemental est associé à l'utilisation des technologies de l'information et de la communication de manière générale. La transaction en ligne peut toujours être compromise par un tiers qui peut récupérer frauduleusement les données personnelles ou utiliser les coordonnées bancaires à l'insu de la volonté du client..., et ce, malgré toutes les mesures de sécurisation des transactions mises en place par les sites marchands.

\subsection{Les Déterminants De La Confiance En Ligne}

La revue de la littérature théorique et empirique sur la confiance en l'achat a permis d'identifier ses principaux déterminants. Ils peuvent être répartis en trois catégories: La première est liée aux caractéristiques du site web, la seconde aux variables sur le commerçant en ligne, la troisième aux variables de contexte à savoir le risque perçu.

\subsubsection{Les variables liées au site marchand}

Les effets de l'interface d'un site web sur les intentions et décisions d'achat ont fait l'objet de nombreuses études en marketing (Jarvenpaa et al., 2000 ; Hu et al., 2010). Selon McKnight et al. (2002), les impressions du consommateur se forment sur la base de l'interface web «vitrine » de la marque. Les attributs d'un site web auraient un impact direct sur les craintes de l'internaute et ses réticences quant à l'enclenchement du processus de l'achat en ligne. Ces attributs concernent la facilité d'utilisation, l'ergonomie du site et la sécurité de la vie privée (Suh et Han, 2003 ; Chang et Chen, 2008 ; Demangeot et Broderick, 2007).

L'ergonomie du site et sa facilité d'utilisation : l'ergonomie illustre l'ensemble des caractéristiques graphiques créant une atmosphère conviviale et attractive pour le consommateur (Moore et al., 2005 ; Hussin et al., 2006 ; Stremtan et Muntean, 2008). La facilité d'utilisation, quant à elle, évoque le degré d'aisance et de commodité du site marchand. Pour Wang et Emurian (2004), la simplicité d'un site et la cohérence des informations qui y figurent permettent de réduire l'appréhension du consommateur et du risque perçu à l'égard de ce site. L'accessibilité à l'information utile est également une dimension importante. Benbasat et Wang (2005) ont proposé que la confiance en l'achat en ligne est engendrée par six facteurs dont trois sont liés aux 
interactions fluides : la navigation, la réalisation (le moment à partir duquel le processus d'achat est initié jusqu'à la réception du produit) et la technologie induisant la fonctionnalité et la vitesse.

La facilité d'utilisation perçue est également présente dans le modèle d'acceptation technologique (TAM; Technology Acceptance Model) de Davis (1989). En effet, Davis (1989) suggère que l'acceptation d'une technologie nouvelle est déterminée par deux facteurs : l'utilité perçue du système et sa facilité d'usage perçue. L'utilité perçue renvoie au degré de croyance du potentiel de performance et de bénéfice de l'utilisation qu'une personne accorde à une technologie. La facilité d'usage perçue, quant à elle, se réfère au degré auquel une personne croit que l'utilisation d'un système sera dénuée d'efforts. Ainsi, face à deux systèmes offrant les mêmes fonctionnalités, un utilisateur aura tendance à choisir le plus facile à utiliser et aussi le plus utile (Dillon et Morris, 1999).

La perception de vie privée et de sécurité en ligne : Les menaces à la sécurité sont définies comme étant «circonstance, situation ou événement susceptible de causer des difficultés économiques aux données ou aux ressources du réseau sous forme de destruction, divulgation, modification de données, déni de service et / ou fraude, gaspillage, et abus » (Belanger et al., 2002). L'altération de la sécurité peut être due à toutes manipulations d'informations sans consentement du consommateur causant, modifications et falsifications des données personnelles, non-respect de l'engagement avec pertes monétaires ou non monétaires (Halaweh, 2012). Les questions de sécurité et confidentialité ont une influence élevée sur le comportement des consommateurs (Miyazaki et Fernandez, 2001 ; Chellappa, 2002) et ont un impact sur la confiance (Suh et Han, 2003 ; Corbitt, 2003 ; Kim et al., 2009). Ces derniers s'accordent à dire que la sécurité perçue d'un consommateur est moindre en ligne qu'hors ligne.

\subsubsection{Les variables liées au marchand}

Une des principales dimensions de la confiance réside dans les attributs du vendeur, selon Jarvenpaa et Tractinsky (1999) «la confiance ne peut exister que si le consommateur croit que le vendeur a à la fois la capacité et la motivation de livrer des biens et services dans la qualité attendue ». La littérature théorique et empirique a identifié plusieurs caractéristiques permettant de renforcer la croyance de confiance et la perception de fiabilité (McKnight et al. (2001), à savoir l'intégrité, la crédibilité et la réputation.

La réputation est définie par McKnight et al. (2001) comme «les caractéristiques qu'on attribue à une personne ou à une organisation sur la base d'informations de seconde main ». Cette définition est particulièrement pertinente dans le cas de la confiance initiale où le client n'a pas d'historique d'expériences passées avec un fournisseur. En effet, sans interactions préalables, le client ne peut juger de la crédibilité et de la fiabilité du vendeur 
(Kamari, F. et Kamari, S. ; 2012). L'opinion de précédents clients peut impacter positivement la confiance de futurs utilisateurs et peut enclencher le processus intentionnel d'achat (Nguyen et Leblanc, 2001). Par la suite, la prise de décision est facilitée car la réputation est un actif distinct, non reproductible et un atout concurrentiel (Rajaobelina et al., 2011).

La réputation peut être construite et exige un investissement à long terme en ressources financières et publicitaires (Jarvenpaa et al., 2000). Pour Kamari, F. et Kamari, S. (2012), elle peut être bâtit de deux manières, en premier lieu, par de l'auto-réputation via de la publicité notamment dans les portails web les plus visités. Certains sites web affichent sur leur page les témoignages de leurs clients ou anciens utilisateurs pour renforcer leur réputation (Jarvenpaa et al., 2000). Ces méthodes sont assez répandues chez les sites de ventes exclusives en ligne tels qu'Amazon qui utilisent les commentaires et la notation pour rassurer les consommateurs sur certains produits. En second lieu, la réputation peut être construite via les comparateurs de produits et services, qui sont plus crédibles que l'autopromotion car générés par des tiers.

La crédibilité Selon Ltifi et Gharbi (2012) représente l'appréciation par le consommateur des capacités techniques du vendeur à remplir les termes de la transaction et à répondre à ces besoins spécifiques (Bart et al., 2005). Elle fait référence au niveau d'expertise technique, organisationnelle et managériale du marchand en ligne. La crédibilité peut être évaluée par la présentation des produits et des informations les concernant (Papadopoulou et Martakos, 2008). Le gain de temps constitue un gage de crédibilité. Les consommateurs sont davantage enclins à poursuivre les échanges avec une entreprise qui garantit l'accès rapide et facile aux produits et services. En outre, le respect des délais de livraison des produits ou services est une dimension de la crédibilité (Gurviez et Korchia, 2002 ; Corritore et al., 2003). Les produits livrés doivent être conformes aux attentes du consommateur et aux termes de l'échange. Un autre indicateur de performance est celui des prix des produits qui doit être concurrentiel (Wang et Emurian, 2004; Donada et Nogatchewsky, 2007).

L'intégrité est avant tout une qualité humaine, celle de communiquer et d'agir honnêtement et avec justice. Elle est représentée par Ltifi et Gharbi (2012) comme «l'attribution des motivations loyales et fiables à la marque quant au respect de ses promesses concernant les termes de l'échange, autrement dit de l'honnêteté de son " discours " pris au sens large». L'intégrité est donc la manifestation du respect des promesses du vendeur envers le consommateur (Chouk et Perrien, 2005), et ce, en termes de délais de livraison et de conformité à la commande effectuée par le client. Papadopoulou et Martakos (2008) dans leur modèle (testé empiriquement auprès de 132 universitaires) sur les étapes de la formation de la confiance en 
ligne, ont attesté que l'intégrité était la troisième évaluation générant la confiance des clients après la bienveillance et la compétence. La bienveillance est l'orientation du fournisseur à résoudre les problèmes et à pérenniser la relation avec les clients (Hess et Story, 2005). Cette dimension est particulièrement importante dans le e-commerce car elle permet de réduire les incertitudes perçues du consommateur (Allagui et Temessek, 2005 ; Ltifi et Gharbi, 2012).

\subsubsection{Variables liées au consommateur}

Dans ce cadre, trois dimensions importantes peuvent être relevées dans la littérature : la familiarité avec le site et degré d'expertise de l'internaute et la propension à faire confiance.

La familiarité avec le site et le degré d'expertise de l'internaute : La familiarité peut être définie comme un ensemble d'apprentissages générés par le biais d'interactions et d'expériences (Gefen, 2000). La familiarité avec un site web notamment par l'expérience préalable d'achat a été dans nombres d'études associée au risque perçu et à l'intention d'achat (McKnight et al., 2002 ; Beneke et al., 2010). En effet, les expériences passées permettent de fournir les connaissances nécessaires sur les moyens techniques dont dispose le site web et l'assurance de l'importance qu'il accorde au niveau de la sécurité (Halaweh, 2012). La familiarité permet de diminuer la complexité et réduit l'incertitude par l'établissement d'un cadre structurel de fiabilité (Gefen, 2000). Pour Gefen (2000), la familiarité est la condition préalable de la confiance car elle permet de créer un cadre futur favorable et qu'en même temps elle peut anéantir la confiance en cas de mauvaise expérience. Cette hypothèse a été validée par Pavlou (2003), la répétition des transactions en ligne avec un fournisseur est tributaire de la satisfaction antérieure. Dans la même veine, Yoh (2003) montre que l'expérience antérieure influence positivement l'intention d'achat des vêtements en ligne.

Par ailleurs, l'expertise du consommateur est un concept distinct de la familiarité car il fait appel au degré de connaissances dans l'utilisation d'Internet en général et des sites web commerciaux indépendamment de l'expérience antérieure avec un fournisseur en particulier. Plusieurs études empiriques soutiennent que les individus possédant un certain niveau d'expertise d'Internet sont plus à même à faire confiance dans les vendeurs en ligne (Gefen, 2000 ; Bart et al., 2005 ; Chang et Fang, 2013). En effet, les utilisateurs avertis ont plus d'aisance à naviguer dans les sites web, à trouver les informations recherchées et ont tendance à maitriser les astuces de l'achat en ligne. Il en va de même de la crédibilité perçue du fournisseur qui est favorablement corrélée au niveau d'expertise de l'internaute (Flanagin et Metzger, 2003 ; Chang et Fang, 2013).

La propension à faire confiance : «faire confiance » ou « ne pas faire confiance» peut être un trait de personnalité chez certains 
consommateurs (Lee et Turban, 2001 ; Chang et Fang, 2013). Comme précisé plus haut, la confiance est une variable psychologique liée au comportement des individus. De plus, elle est plus élevée chez certaines personnes que d'autres qui seront initialement plus méfiants (Lee et Turban, 2001). Gefen (2000) la définit comme «une disposition générale, une inclinaison à afficher la foi en l'humanité et à adopter une position de confiance envers les autres ». McKnight et al., (2004) la décrit également comme la volonté de dépendre et de se mettre en situation de vulnérabilité. La propension à faire confiance ne repose pas par ailleurs sur les expériences passées mais elle est plus un mode de vie social (McKnight et al., 2002 ; Chouk et Perrien, 2005). Ganguly et al., (2009) approchent la notion en la comparant à la notion de méfiance. En effet, pour ces auteurs, une personne avec faible propension à faire confiance sera plus critique vers les produits et services en ligne et même envers la perception de la qualité du site web. Ces mêmes auteurs montrent que les consommateurs méfiants seront plus exigeants et attentifs à la réputation des vendeurs, aux commentaires et expériences vécues que la moyenne des consommateurs.

Le lien entre la disposition à faire confiance et la confiance en ligne n'a pas fait l'objet de plusieurs études. Les recherches sur la construction de la confiance installée ont été plus développées au détriment de la confiance initiale. Néanmoins, la plupart des recherches empiriques sur la question de la propension à faire confiance joue un rôle modérateur (Lee et Turban, 2001 ; Chouk et Perrien, 2005). D'autres ont testé la question dans un contexte hors ligne, qui ne peut être appliqué dans le cas du commerce électronique. Plusieurs travaux attestent empiriquement que les individus qui ont une forte propension à faire confiance sont plus disposés à accorder leur confiance dans les sites web commerciaux notamment dans la phase initiale où les consommateurs sont moins familiers avec les vendeurs (Chouk et Perrien, 2005 ; Koufaris et Hampton-Sosa, 2004).

\subsubsection{Variable liée au contexte : Le risque perçu}

Le risque perçu est une dimension à part entière de la confiance en ligne. Il est défini comme l'incertitude sur les effets indésirables et les conséquences de la prise d'une décision (Chouk et Perrien, 2005). Mitchell (1999) a suggéré que le risque perçu était une variable décisive pour expliquer le comportement des consommateurs et que leur niveau de perception dépendait d'un consommateur à l'autre.

En ligne, le risque perçu est le premier obstacle à l'adoption du commerce électronique, les études ont révélé que la perception du risque perçu associée aux pertes possibles découlant de la transaction en ligne est plus élevée chez les internautes que chez les consommateurs dans les environnements traditionnels. Le risque perçu est un construit ayant une influence en deux temps, son effet est élevé au moment de l'évaluation des alternatives et de la décision d'achat, que dans les étapes antérieures de 
reconnaissance du besoin et de recherche d'information (Bèzes, 2011). Cette définition montre que le consommateur prête attention davantage à l'incertitude relative aux conséquences (de manière subjective) que sur les conséquences elles-mêmes (Chouk et Perrien, 2005).

Pavlou (2003) a classé deux catégories d'incertitude présentes dans les transactions en ligne: une incertitude comportementale et une incertitude générale. La première est liée au risque que le marchand en ligne adopte un comportement opportuniste en prenant avantage de la nature lointaine et impersonnelle du commerce électronique et du manque de régulation visant à protéger l'internaute. Les types de risque perçu liés à cette incertitude ont été classés par plusieurs auteurs (Gefen, 2000 ; Grazioli et Jarvenpaa, 2000 ; McKnight, 2002 ; Kim et al., 2009). La typologie et la hiérarchie des risques perçus pour le commerce électronique est pratiquement similaire à celle du commerce physique (Forsythe et Shi, 2003). Les différents types de risques identifiés étaient relatifs à l'objet physique, au temps, à la sécurité, à la dimension de la performance du marchand en ligne (Forsythe et Shi, 2003). Bhatnagar et al. (2005) ont mentionné trois principaux types de risques prédominant dans l'achat en ligne ; le risque financier, le risque lié au produit et le risque lié à l'information (sécurité et confidentialité).

Le schéma ci-après résume les principaux déterminants de la confiance en l'achat en ligne relevés dans la littérature :

Schéma 1 : Les déterminants de la confiance en l'achat en ligne

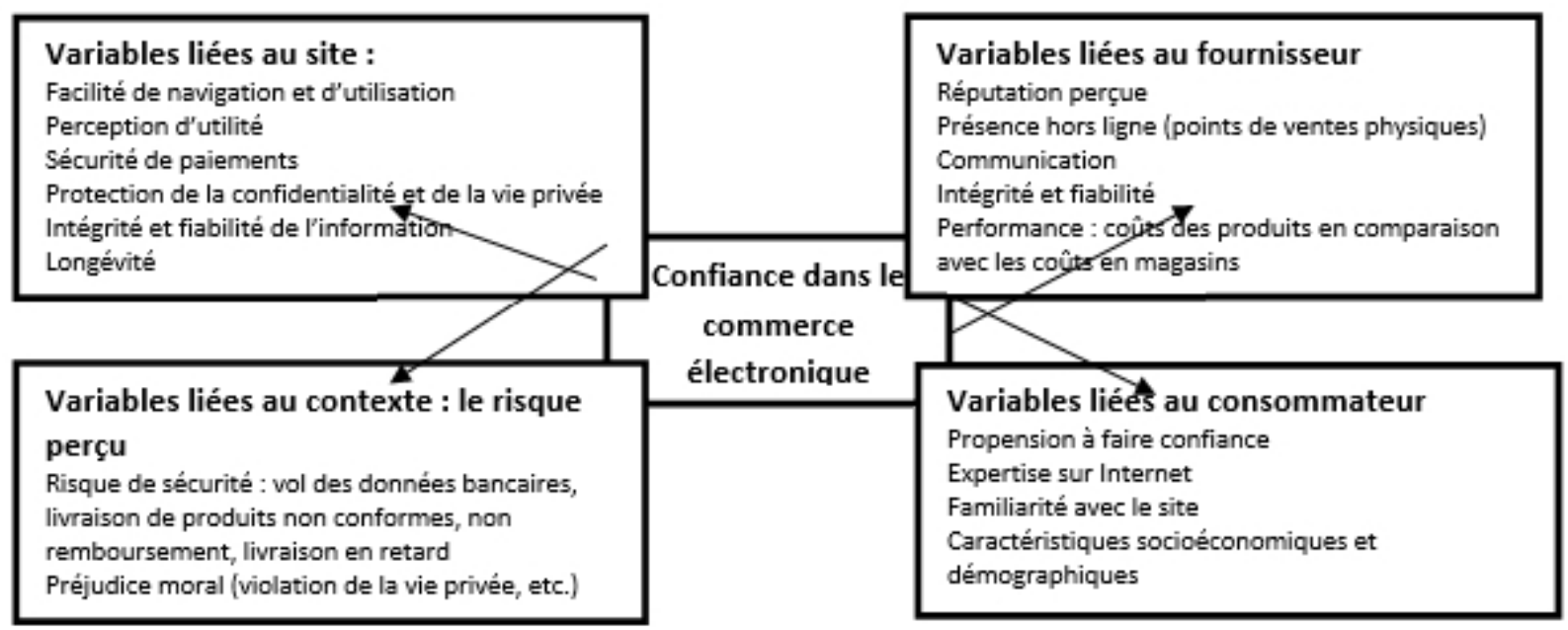

Après avoir passé en revue les principaux déterminants théoriques et empiriques de la confiance en l'achat en ligne, la section suivante présente brièvement l'enquête ainsi que les principaux résultats. 


\section{Résultats De L’enquête : Méthodologie, Résultats A Plat Et Des Analyses En Composantes Multiples}

Afin de déceler empiriquement les déterminants de la confiance en l'achat en ligne au Maroc, un questionnaire original a été administré par faceto-face auprès d'un échantillon représentatif de 2254 individus localisés dans trois villes marocaines différentes en termes des conditions économiques et sociodémographiques des individus : la métropole de Casablanca, la ville de Meknès et la commune urbaine de Laâyoune orientale. Ce choix méthodologique peut être justifié par la volonté de déceler de potentielles divergences de comportement envers le commerce électronique selon les conditions économiques locales et selon le profil socioéconomique des individus.

\subsection{L'échantillonnage et premiers résultats exploratoires}

Du fait de l'absence d'une liste exhaustive et fiable de la base de sondage, on a décidé d'opter pour l'échantillonnage empirique en y introduisant de l'aléatoire afin de limiter les risques de biais. Ainsi, les enquêtes ont été effectuées «au pas » en interrogeant arbitrairement une personne sur vingt rencontrés. De plus, les personnes ont été interrogées à des heures différentes de la journée et dans des endroits différents. 2254 réponses ont été recueillies réparties comme suit : 934 répondants à la ville de Casablanca (41\% de l'échantillon), 711 à la ville de Meknès (32\% de l'échantillon) et 609 à la ville de Laayoune orientale (27\% de l'échantillon).

Les premiers résultats à plat de l'enquête font ressortir que, toutes villes confondues, $88 \%$ des répondants de l'échantillon déclarent avoir une connexion à Internet (graphique 1). Ce résultat est dû à la démocratisation de l'Internet $3 \mathrm{G} / 4 \mathrm{G}$ qui assure normalement un accès Internet à toute personne possédant un téléphone portable intelligent. Ce taux cache tout de même une certaine disparité entre les trois villes dans la mesure où le taux d'accès à la technologie est de $93 \%$ à Casablanca contre $81 \%$ pour Laayoune Orientale.

Graphique 1. Taux de pénétration d'Internet

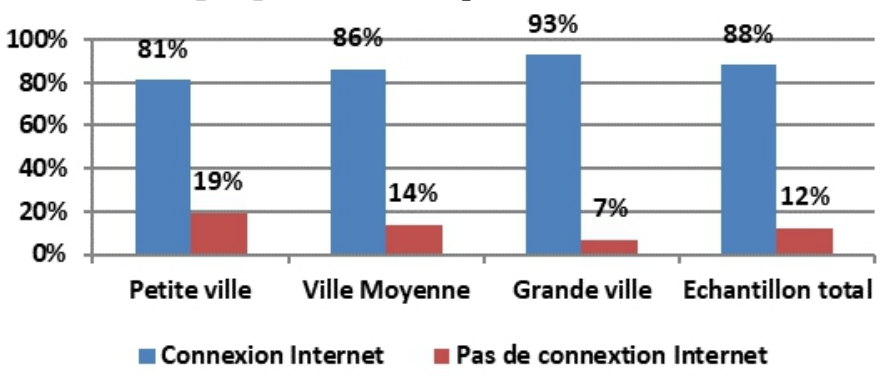

Pour ce qui est du commerce en ligne, $34 \%$ des individus de l'échantillon total déclarent avoir déjà effectué au moins un achat en ligne au cours des 12 derniers mois. Le taux du E-achat semble être corrélé 
positivement à la dimension de la ville. En effet, il passe de $27 \%$ pour la petite ville à $31 \%$ pour la ville moyenne et à $40 \%$ pour la métropole de Casablanca. Les raisons principales qui sous-tendent l'achat en ligne (graphique ${ }^{\circ} 2$ ) et qui sont partagées largement par les répondants des trois villes sont «L'achat sur Internet facilite la comparaison des offres » $(93 \%)$; «L'achat sur internet m'évite des déplacements » $(89 \%)$; «L'achat sur Internet représente un gain de temps » $(85 \%)$; «L'achat sur Internet facilite la comparaison des prix » (79\%) ; « Je trouve sur Internet de nouveaux types de produits et de services innovants » $(77 \%)$; «L'achat sur Internet permet de bénéficier de prix plus avantageux et de promotions » $(74 \%)$ et «Il y a plus de choix sur Internet qu'en magasin » $(67 \%)$.

Graphique $\mathbf{n}^{\circ} 2$ : Les avantages de l'achat en ligne

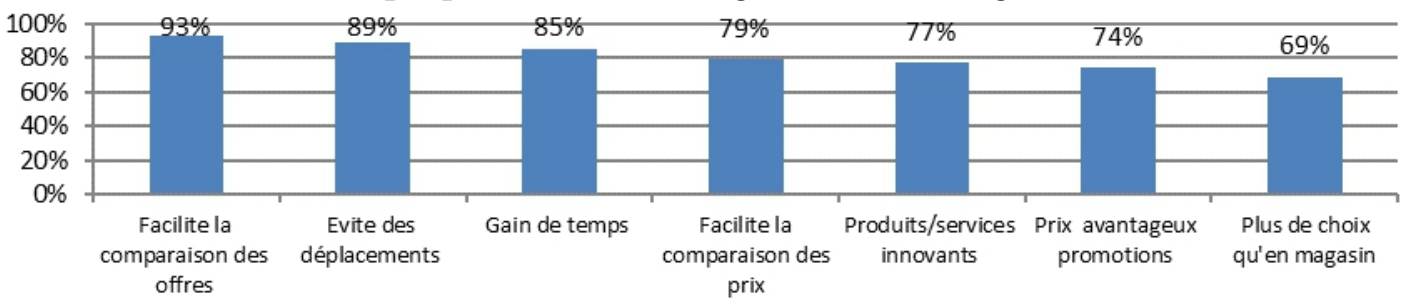

En revanche, les raisons du non-achat en ligne (graphique 3) semblent être clairement liées à un problème de méfiance vis-à-vis du E-commerce, et ce, quelle que soit la ville de résidence. En effet, $54 \%$ des répondants de l'échantillon total affirment refuser d'effectuer des achats sur internet car ils n'ont pas confiance $(56 \%$ pour Casablanca, $51 \%$ pour Meknès et $53 \%$ pour la petite ville de Laayoune Orientale). De même, $48 \%$ des personnes interrogées déclarent préférer acheter leurs produits en magasin. Le troisième frein au commerce en ligne cité par les répondants est la non-détention d'un compte bancaire à hauteur de $34 \%$. De même, pour $18 \%$ des répondants de l'échantillon total et des villes de Meknès et de Casablanca, le refus d'achat en ligne serait lié à la complexité de la procédure dans la mesure où ils avancent «ne pas savoir comment faire pour effectuer à un achat en ligne ». Ce résultat corrobore les résultats de Wang et Emurian (2004) qui ont démontré que la simplicité d'un site et la cohérence des informations qui y figurent permettent de réduire l'appréhension du consommateur et du risque perçu à l'égard de ce site. Les habitants de la petite ville, quant à eux, placent en quatrième position le frein relatif au non-accès à internet à raison de $24 \%$. A ce titre, la non-adoption de l'achat en ligne par la ville périphérique serait expliquée par la fracture numérique de premier degré dont l'origine relève de la non-accessibilité au numérique. 
Graphique $\mathbf{n}^{\circ} 3$ : Les freins à l'adoption du E-commerce

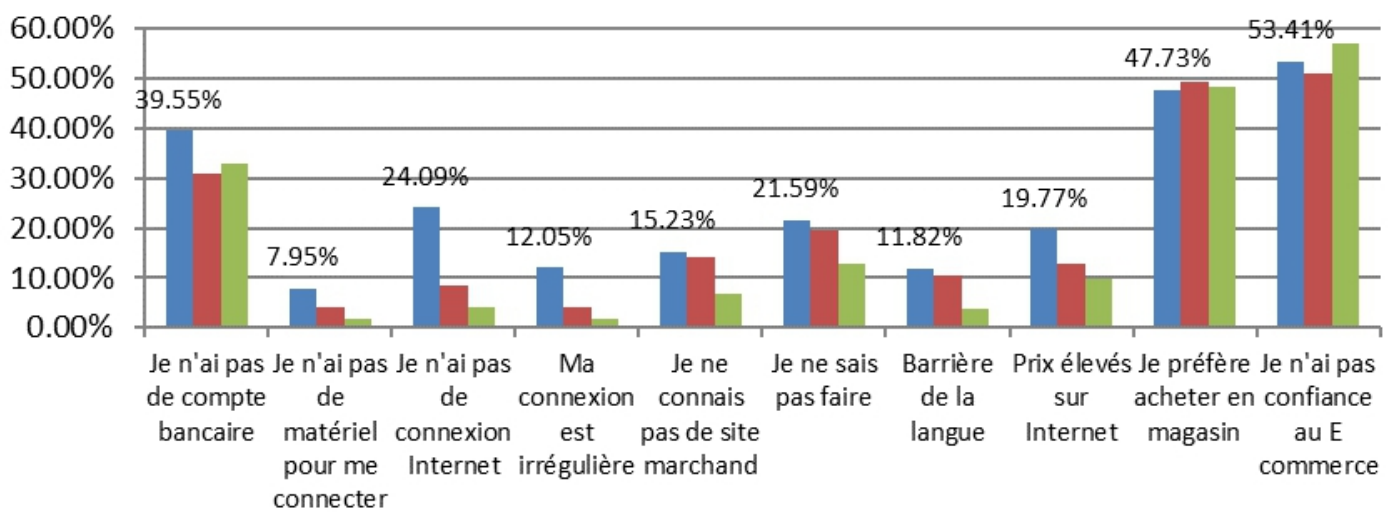

Petite ville $\quad$ Ville moyenne $\quad$ Grande ville

Ces résultats à plat amènent naturellement vers des traitements statistiques multidimensionnels. L'objectif est de pouvoir extraire les variables qui expliquent la propension en la confiance en l'achat en ligne par les habitants des trois villes. On a effectué une ACM regroupant l'ensemble des variables et des individus mais en étiquetant les individus selon la variable « localisation ». L'objectif est de vérifier si cette variable sera active dans la construction des axes mais aussi de déceler des différences de comportement des individus selon leur lieu de localisation.

\subsection{Résultats Des Analyses En Composantes Multiples (ACM)}

La statistique descriptive multidimensionnelle regroupe l'ensemble des méthodes de la statistique exploratoire dont l'objectif est le traitement simultané de plusieurs variables. Ces méthodes, étant essentiellement descriptives, ne font référence à aucun modèle de nature probabiliste. La méthode la plus utilisée de la statistique descriptive multidimensionnelle est l'analyse factorielle. Elle consiste à rechercher des « facteurs » en nombre limité résumant le mieux possible les données considérées. Elle permet de représenter géométriquement par des graphiques, généralement des nuages de point ou diagramme de dispersion, les rapprochements et les divergences entre les individus selon diverses caractéristiques ou variables. Sur ces graphiques, les individus statistiques qui se ressemblent (tout comme les modalités partagées par les mêmes individus) sont représentés par des points qui tendent à se regrouper; la dissemblance produit, au contraire, de la distance.

Il existe plusieurs techniques d'analyse factorielle qui dépendent du type de données auquel est confronté le statisticien. Les plus courantes sont l'analyse en composante principale (ACP) portant sur des variables quantitatives, l'analyse factorielle des correspondances (AFC) portant sur 
deux variables qualitatives et l'analyse des correspondances multiples (ACM) portant sur plusieurs variables qualitatives. Pour cette étude, la nature des données recueillies et la problématique traitée amènent à utiliser l'Analyse en Correspondances Multiples.

\subsubsection{Note méthodologique sur l'Analyse des Correspondances Multiples \\ L'analyse des Correspondances Multiples (ACM), est une méthode} factorielle de statistique descriptive multidimensionnelle permettant de traiter simultanément un nombre quelconque de variables. Elle est adaptée aux données issues d'enquêtes d'opinion dans lesquelles les répondants ou les individus (en lignes) sont décrits par un ensemble de variables qualitatives (en colonne). L'ACM est souvent utilisée dans les dépouillements d'enquêtes, lorsqu'on souhaite aller au-delà des simples tris à plat. L'ACM permet de résumer l'information contenue dans un grand nombre de variables afin de faciliter l'interprétation des corrélations existantes entre elles. Elle permet d'aboutir à des cartes de représentation sur lesquelles on peut visuellement observer les proximités entre les catégories des variables qualitatives et les observations. La projection orthogonale du nuage de point sur un espace à deux dimensions permet d'avoir une meilleure approximation des données que par les tris à plats et les tris croisés. Le premier axe est celui qui résume les corrélations entre les variables. Le second axe, quant à lui, résumera le mieux le reste de l'information. Pour chaque axe, on calcule la part d'inertie de l'axe exprimée en pourcentage. Elle exprime la quantité d'information que résume chaque axe.

Par ailleurs, l'ACM permet également de renseigner sur les contributions des différentes modalités de chacune des variables à la détermination des axes. Si la contribution est proche de zéro, la modalité n'exerce que très peu ou pas d'influence. Si la contribution est forte ${ }^{10}$, la modalité exerce une influence sur les axes. Cette influence peut être positive ou négative selon le signe de sa coordonnée sur les axes.

D'un point de vue méthodologique, l'ACM est une Analyse Factorielle des Correspondances réalisée sur un tableau de Burt relatif à plusieurs variables qualitatives (au moins trois). Le tableau de Burt, qui est une table symétrique, est une généralisation de la table de contingence pour un nombre $\boldsymbol{v}$ de variables qualitatives. C'est une matrice carrée constituée de $\mathbf{v}^{2}$ sousmatrices. Chacune des sous-matrices diagonales est relative à l'une des $v$ variables. Il s'agit donc d'étudier les liaisons susceptibles d'exister entre les $v$ variables considérées ou des proximités/distances entre des modalités de variables différentes ou entre individus.

\footnotetext{
${ }^{10}$ Quand elle dépasse la moyenne des contributions de l'ensemble des modalités.
} 
L'ACM passe dans un premier lieu par une analyse discriminante, utilisée pour mesurer le pouvoir discriminant d'une variable relativement à une dimension. Ce dernier est défini comme le pourcentage de variance de la dimension expliquée par cette variable. L'étape qui suivra la définition des dimensions, consistera à caractériser les relations entre les modalités d'une même variable, mais aussi entre celles de variables distinctes, en situant chaque modalité sur le même graphique au moyen de leurs coordonnées factorielles selon chaque dimension.

Etant donné que l'enquête a été réalisée par «face to face», le questionnaire a été programmé sur le logiciel de traitement d'enquête Sphinx afin de pouvoir saisir facilement les réponses et récupérer la base de données. En revanche, l'analyse factorielle a été effectuée par le logiciel SPSS.21 largement utilisé pour l'analyse statistique des données dans les sciences sociales. Le traitement effectué a abouti aux résultats présentés ci-dessous.

\subsubsection{Résultat de l'ACM globale}

Dans cette ACM, on a intégré toutes les variables et tous les individus de l'échantillon. On a étiqueté les individus dans le nuage des points-individus selon la variable localisation pour voir l'impact qu'elle aura sur la définition des deux dimensions mais aussi sur le comportement des répondants.

- La discrimination par les variables

Le premier tableau (tableau $\mathrm{n}^{\circ} 1$ ) indique que les variables sélectionnées ont dégagé deux facteurs. Ils représentent $42 \%$ de l'inertie totale. Rappelons que l'inertie d'un axe mesure la liaison entre l'axe et les variables. Elle est égale à la moyenne des carrés des rapports de corrélation entre l'axe et les variables. Le pourcentage d'inertie peut être relativement faible car les individus évoluent dans un espace de dimensions élevées, d'autant plus élevé que le nombre de modalités par variable est grand. Pour cette étude, le pourcentage d'inertie est de l'ordre de $42 \%$. Il est plus que satisfaisant car les deux facteurs résument à eux seuls $42 \%$ de l'ensemble des informations données par l'ensemble des variables introduites.

La deuxième statistique intéressante de ce tableau est l'Alpha de Cronbach (1957), appelé aussi coefficient de consistance interne. C'est une mesure fréquemment utilisée lorsque l'on traite des données subjectives issues d'enquêtes d'opinion. Ainsi, l'alpha de Cronbach permet de mesurer la fiabilité ou la cohérence interne d'un ensemble d'items censés contribuer à mesurer un phénomène commun. Le calcul de cet indice présume une forte corrélation entre les items. C'est la raison pour laquelle un alpha de Cronbach dont la valeur se rapproche de 1 dénote une cohérence interne parfaite entre les items tandis qu'une valeur proche ou égale à 0 ne justifie aucune cohérence interne entre les items en question. En pratique, on considère que l'alpha de Cronbach doit supérieur à 0,7 pour que la consistance interne soit justifiée. 
Pour cette étude, l'ACM présente un alpha de Cronbach de 94\%, ce qui est excellent car il se rapproche de 1 et dépasse le seuil minimum requis de $70 \%$. Il dénote d'une très bonne cohérence interne et que les variables mesurent le même construit.

Tableau 1 : Représentativité des variables

\begin{tabular}{|c|c|c|r|r|}
\hline \multicolumn{5}{|c|}{ Récapitulatif des modèles } \\
\hline \multirow{2}{*}{ Dimension } & \multirow{2}{*}{$\begin{array}{c}\text { Alpha de } \\
\text { Cronbach }\end{array}$} & $\begin{array}{c}\text { Total (valeur } \\
\text { propre) }\end{array}$ & Inertie & $\begin{array}{c}\text { Pourcentage } \\
\text { de variance } \\
\text { expliquée }\end{array}$ \\
\cline { 3 - 5 } & & 22,776 & 0,340 & 33,994 \\
\hline 1 & 0,971 & 5,716 & 0,085 & 8,532 \\
\hline 2 & 0,838 & 28,493 & $\mathbf{0 , 4 2 5}$ & \\
\hline Total & & 14,246 & 0,213 & 21,263 \\
\hline Moyenne & $\mathbf{0 , 9 4 4}^{\text {a }}$ & & & \\
\hline
\end{tabular}

Avant de passer à l'interprétation proprement dite, il faut s'intéresser à la contribution du couple (individus, modalités) à l'inertie des facteurs. S'impose alors l'étude comparative des tableaux synthétiques présentant, facteur par facteur, les contributions des individus et des modalités, éventuellement groupées par variables.

Le tableau de mesure de discrimination renseigne sur la corrélation de chaque variable avec chacun des deux axes. Il donne les mesures de discrimination des variables sur les axes. on remarque que c'est la variable Eachat qui discrimine le mieux les individus sur le premier axe avec un taux de corrélation exceptionnelle de 90,7\%. Plusieurs variables contribuent à plus de $80 \%$ à la définition de ce premier axe notamment celles ayant trait aux avantages du commerce en ligne tels qu'ils sont perçus par les répondants. Ces variables sont les suivantes : "l'achat en ligne constitue pour moi un gain de temps »; «l'achat en ligne permet de limiter les déplacements en ville »; «l'achat en ligne me permet de faire du shopping à n'importe quelle heure »; «l'achat en ligne permet la comparaison des offres sur Internet »; «l'achat en ligne permet de profiter des promotions qu'on ne trouve pas dans les magasins »; «l'achat en ligne permet de comparer les prix facilement »; « je trouve sur internet de nouveaux types de produits et de services innovants»; « il y'a plus de choix sur internet qu'en magasin »; « le catalogue des produits en ligne est attractif »; «le degré de difficulté de l'achat en ligne » et «l'intention d'effectuer des achats en ligne à l'avenir ».

Un autre groupe de variables discriminant à plus de $70 \%$ les individus sur le premier axe sont celles liées à la confiance des consommateurs en l'achat en ligne. Il s'agit des variables suivantes : «Je n'ai pas confiance même si le paiement est à la livraison»; «Absence de législation»; «les données personnelles ne sont pas protégées sur internet »; «c'est dangereux de donner son numéro de carte bleue sur internet»; «certains vendeurs ne sont pas 
honnête »; «j'ai peur d'être arnaqué ou de subir une tremperie de la part du vendeurs »; « je préfère toucher avant d'acheter le produit »; « «j'ai peur que le produit ne corresponde pas »; «j'ai peur de ne pas me faire livrer»; «les services après vente et l'assistance ne sont pas satisfaisants $» ;\langle\mathrm{j}$ 'ai peur de ne pas me faire rembourser ».

La variable ville ou lieu de résidence présente un taux de corrélation très bas avec les deux axes (1,2\% avec le premier et $9,4 \%$ avec le second) et a par conséquent un pouvoir discriminatif très faible. Cette ACM globale devra être complétée dans un travail ultérieur par trois ACM spécifiques à chaque ville afin de vérifier la prédominance de chaque variable dans la discrimination des axes.

Pour le deuxième axe, on a relevé que les variables discriminant entre les individus présentent des taux de corrélations relativement faibles par rapport à ce qu'on a observé pour le premier axe. On a retenu les variables contribuant à plus de $20 \%$ classées comme suit par ordre décroissant selon leur contribution à l'axe 2 : la fréquence de connexion à internet $(37,4 \%)$, le niveau d'études $(30,8 \%)$, la catégorie socioprofessionnelle $(26,9 \%)$, regarder des films en streaming $(26,8 \%)$, l'accès à internet $(24,4 \%)$, se former par internet $(24,3 \%)$, se connecter sur les réseaux sociaux $(21,2 \%)$, télécharger des logiciels $(21,2 \%)$, l'âge $(21,9 \%)$ et consulter le courrier électronique $(20,2 \%)$. A travers ces variables, il a été constaté que le facteur 2 est en grande partie déterminé par deux groupes de variables que l'on a classé en trois catégories :

- Les variables socioéconomiques des individus: niveau d'études, catégorie socioprofessionnelle et âge ;

- Les variables liées à l'accès à internet reflétant la fracture numérique de premier degré. L'individu n'achète pas en ligne par contrainte à cause des inégalités économiques et sociales liées à l'accès aux équipements et aux infrastructures ;

- Les variables liées à l'usage de la technologie. L'ACM a révélé que ce sont les usages les plus basiques d'internet qui contribuent le plus à la détermination du deuxième axe. Cela renvoie à la problématique de la fracture numérique de deuxième degré qui reflète les inégalités dans les usages des TIC plus que celles portant sur les inégalités d'accès. En effet, plusieurs travaux théoriques et empiriques effectués dans différents contextes (Hargittai, 2002; Le Guel et al., 2004; Benyoussef, 2004 ; etc.) ont démontré que l'accès à la technologie ne signifie pas automatiquement son usage. L'explication se trouve dans les capacités cognitives des individus qui ne les prédisposeraient pas à avoir un usage efficace et efficient des nouvelles technologies.

Les résultats de mesure de discrimination sont présentés sous forme de diagramme (diagramme $\mathrm{n}^{\circ} 1$ ). Ce diagramme de mesure de discrimination 
indique que la dimension 1 est constituée par une synthèse de variables liées au comportement d'achat en ligne : l'ensemble de ces variables présentent des niveaux d'indice de discrimination importants pour la dimension 1 et faibles pour la dimension 2 . Ainsi, les catégories de ces variables sont bien dispersées selon la dimension 1 et peu dispersées selon la dimension 2. Ceci reflète une distinction selon la dimension 1, sur le diagramme des individus (diagramme $\mathrm{n}^{\circ} 2$ ), entre les cyberconsommateurs confiants, c'est-à-dire les individus ayant l'habitude d'acheter sur internet tous types de biens et services et qui tirent profit de tous les avantages que leur offre ce mode de consommation (situés dans la partie droite du diagramme, D1 $>0$ ) et l'ensemble des autres individus (situés dans la partie gauche du diagramme, D1<0) qui sont méfiants vis-à-vis de l'achat en ligne.

Pour ce qui est du deuxième axe, sont présentées les variables qui présentent des valeurs élevées de l'indice de discrimination selon la dimension 2 , et des valeurs faibles pour la dimension 1 . En conséquence, l'angle entre les vecteurs correspondant à ces variables et la dimension 2 est faible. Cet indice, assimilable au carré d'un coefficient de corrélation $\left(\mathrm{R}^{2}\right)$, exprime la similarité entre lesdites directions. Ainsi, le diagramme de mesure de discrimination montre que la dimension 2 est constituée par des variables liées à l'accès et à la fréquence de connexion à la technologie internet, à certains usages rudimentaires d'internet et à des variables socioéconomiques qui sont le niveau d'études, la catégorie socioprofessionnelle et l'âge. L'analyse du diagramme des individus (diagramme $n^{\circ} 2$ ) relève la distinction observée selon la dimension 2 entre ceux qui achètent en ligne situés dans la partie supérieure $(\mathrm{D} 2>0)$ et l'ensemble des autres individus situés dans la partie inférieure $(\mathrm{D} 2<0)$.

Notons que les variables qui sont concentrées près de l'origine du graphique n'apparaissent pas comme discriminantes dans ce plan à deux dimensions. 
Digramme 1 : mesures de discrimination par variable

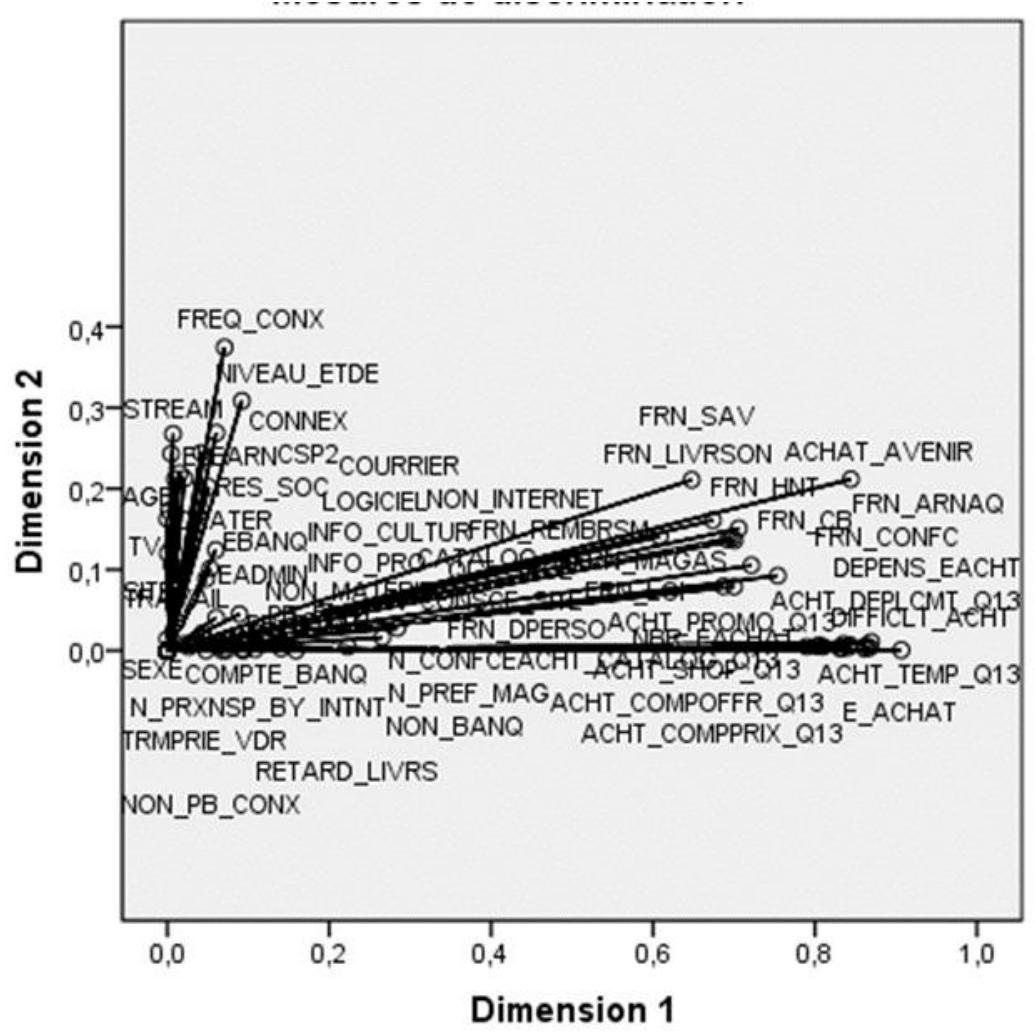

Normalisation principale de la variable.

- La discrimination par les individus

L'analyse de discrimination par variables doit être complétée par celle relative aux individus. La contribution des individus s'avère nécessaire car elle permet de vérifier si un axe n'est pas le résultat d'un très petit nombre de modalités partagées par très peu d'individus. On a fait le choix de présenter le diagramme de discrimination des individus par ville (diagramme $\mathrm{n}^{\circ} 2$ ) dans le sens où chaque individu est étiqueté par son lieu de résidence : 1 pour ceux qui sont localisés dans la petite ville de Laayoune orientale ; 2 pour ceux habitant dans la ville de dimension moyenne Meknès et 3 pour ceux habitant dans la grande métropole de Casablanca. Ce diagramme permettra de visualiser les liens de ressemblances/dissemblances entre groupe d'individus présentant des corrélations entre variables similaires. Ainsi, la distance entre les individus qui partagent exactement le même profil et les mêmes modalités va être égale à zéro. De même, deux groupes d'individus sont ainsi d'autant plus proches que les corrélations entre variables sont semblables d'un groupe à un autre et en particulier pour les variables les plus corrélées entre elles au niveau global. 
Diagramme 2 : mesures de discrimination des individus par ville

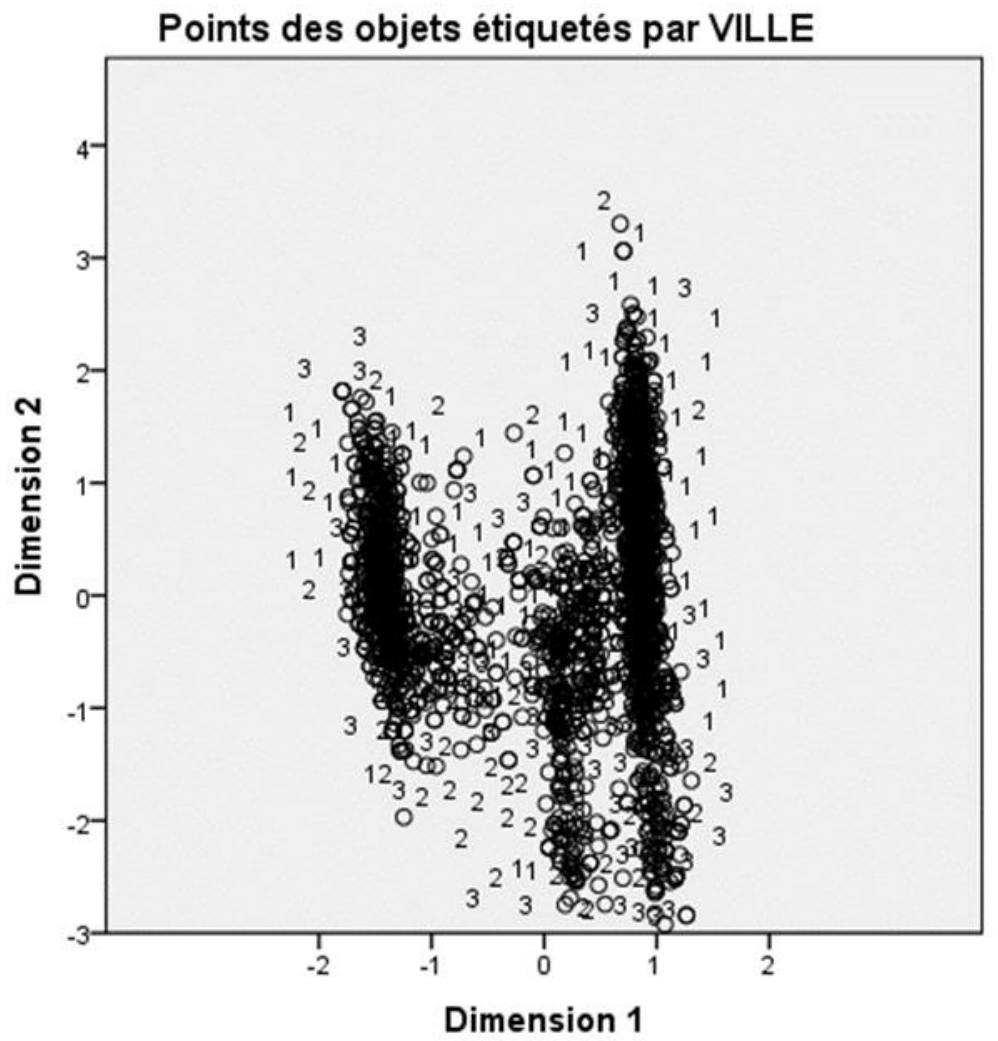

Normalisation principale de la variable.

$1=$ petite ville $; 2=$ moyenne ville $; 3=$ grande ville

Le premier constat de la lecture du diagramme est que la variable lieu de localisation n'est pas déterminante. En effet, la divergence/similitude entre les individus ne semble pas corrélée avec le lieu de résidence. D’ailleurs, ce résultat est conforté par la mesure de discrimination présentée dans le tableau $\mathrm{n}^{\circ} 2$ qui indique un taux de corrélation très faible de la variable « ville de résidence » avec les deux axes ; sa contribution étant de 1,2\% pour la première dimension et de $9,4 \%$ à la deuxième.

La forme du nuage des points par individus permet de mettre en évidence des profils différents selon les variables discriminantes de chaque axe relevées soit à travers le tableau de mesure de discrimination soit par le diagramme $n^{\circ} 2$. Le cadrant droit inférieur du diagramme regroupe les répondants technophiles ayant des usages évolués de l'internet et qui sont des cyberconsommateurs confiants. Ils n'ont aucun à priori vis-à-vis de l'achat en ligne. Autrement dit, ils ont confiance en la technologie et achètent en ligne sans pour autant craindre pour leurs données personnelles, pour leur compte 
bancaire, etc. Ce sont également des personnes ayant un niveau d'études élevés, pour la plupart dépassant le baccalauréat. Cela présuppose qu'ils disposent des capacités cognitives et d'aptitudes leur permettant d'avoir un usage efficace et efficient d'Internet. Ils profitent pleinement de tous les avantages que leur offre l'achat sur internet en termes de diversification des offres, des prix, de l'économie de temps, etc. Ces résultats sont d'autant plus intéressants car la littérature étudiée sur la confiance numérique a toujours pris comme variables liées aux consommateurs la propension à faire confiance et la familiarité avec le site en ligne (Gefen, 2000) et non les caractéristiques sociodémographiques pour expliquer la confiance en ligne.

Les individus concentrés sur la partie droite supérieure ne sont pas forcément regroupés selon la variable E-achat. On y retrouve aussi bien les acheteurs en ligne que les non acheteurs. Le dénominateur commun entre ce groupe de répondants porte sur leur perception commune des freins à l'achat en ligne. Ils ont clairement un problème que l'on qualifiera de « $\underline{\text { méfiance }}$ numérique » liée à l'absence, selon eux, de sécurisation technique et juridique des réseaux numériques et des transactions. A cet égard, les freins qui les réunissent et qui ont dénoté d'une très bonne corrélation avec le premier axe lors de l'analyse discriminante sont : « je n'ai pas confiance même si paiement à la livraison »; « Absence de législation »; « les données personnelles ne sont pas protégées sur internet »; «c'est dangereux de donner son numéro de carte bleue sur internet »; «certains vendeurs ne sont pas honnête »; «j'ai peur d'être arnaqué ou de subir une tremperie de la part du vendeurs »; «je préfère toucher avant d'acheter le produit»; « «j'ai peur que le produit ne corresponde pas »; « j'ai peur de ne pas me faire livrer »; « les services aprèsvente et l'assistance ne sont pas satisfaisants $» ;\langle\mathrm{j}$ 'ai peur de ne pas me faire rembourser ».

Ces résultats corroborent l'hypothèse de l'existence d'une méfiance numérique naturelle envers le e-commerce chez les internautes marocains. La redondance des variables citées plus haut chez les individus questionnés est en accordance avec la littérature sur le risque perçu. La perception du risque perçu associé aux pertes possibles découlant d'une transaction en ligne est plus élevée chez les internautes que chez les consommateurs dans les environnements traditionnels (Grazioli et Jarvenpaa, 2000 ; Gefen, 2000 ; Bhatnagar et al., 2000 ; McKnight et al., 2002 ; Kamari F. et Kamari S., 2012 ).

La partie inférieure gauche du diagramme regroupe les individus qui ne se connectent que peu ou pas du tout et par conséquent n'achètent pas en ligne. Ils ont un niveau d'études inférieur et occupent des emplois manuels ou sont sans activité. Se pose encore une fois la question des connaissances numériques. Beaucoup d'études théoriques et empiriques ont associé le niveau d'instruction à l'utilisation de l'Internet (Warschauer, 2003 ; Singh, 2004 ; 
Oukarfi, 2013). En effet, il est probable que les plus diplômés, qui ont exploité durant leur scolarité les avantages offerts par ces technologies, soient plus portés sur les TIC que les moins diplômés. Cela laisse entendre qu'un niveau d'instruction important permet d'avoir un bagage théorique ou technique favorisant l'usage intensif d'internet. De même, un niveau d'instruction faible ne permet pas d'avoir les aptitudes cognitives nécessaires pour un usage régulier de l'Internet. Dijk (2005) estime que les facteurs intervenant dans l'appropriation des technologies numériques sont les ressources en temps, les ressources sociales et les ressources culturelles qui sont distribuées de manière inégale dans la population. Dès lors, le fait de se retrouver en marge des circuits sociaux, tant éducatifs que professionnels, se révèle être un facteur d'exclusion numérique considérable. Ces premiers résultats tentent à confirmer l'hypothèse la fracture de second degré et la question de l'influence des compétences numériques sur l'adoption de l'achat en ligne.

\section{Discussion}

L'ACM a permis de mettre le voile sur des résultats intéressants conformes aux prédictions théoriques sur les déterminants de la confiance en l'achat en ligne. On a cherché à identifier de potentielles variables prépondérantes dans la construction des facteurs aussi bien du côté des individus que des modalités. Le premier résultat probant obtenu par les mesures de discrimination est que c'est la variable E-achat qui discrimine le mieux les individus sur le premier axe. Elle a présenté un taux d'inertie exceptionnel dépassant les 90\%. D’autres variables se sont révélées déterminantes, toujours pour ce premier axe, notamment celles relatives aux avantages du E-commerce en termes de réduction du temps, des déplacements, de comparaison des offres et des prix, et celles relatives aux freins de ce mode novateur de consommation reflétant une « méfiance » du consommateur visà-vis du numérique en général et du commerce électronique en particulier. D'autres variables ont présenté un taux de corrélation élevée avec le premier axe : la difficulté perçue de l'expérience d'achat en ligne (élevée, moyenne ou faible) et la volonté/refus du consommateur à acheter en ligne dans le futur.

On a ainsi pu relever au moins trois profils d'individus. Les individus technophiles présentant une certaine dépendance vis-à-vis du numérique et qui sont des cyberacheteurs convaincus. Le commerce en ligne représente pour eux un style de vie. En effet, ils ont adhéré à ce mode de consommation pour profiter pleinement de ses avantages en termes de diversification des offres, des prix, de l'économie de temps et d'argent, etc. On les a qualifiés de « cyberacheteurs confiants » car ils n'expriment aucun à priori vis-à-vis du commerce électronique. Plus encore, ils expriment clairement la volonté de continuer à effectuer des achats en ligne. En revanche, on a relevé l'existence de « cyberconsommateurs-méfiants » c'est-à-dire des acheteurs en ligne, 
certes, mais qui expriment tout de même une méfiance à l'égard de l'achat en ligne expliquée par des facteurs exogènes. Ils sont tout de même unanimes sur les risques qu'ils peuvent encourir en adoptant ce mode d'achat. Ils déclarent avoir un problème de méfiance numérique dû à plusieurs facteurs, entre autres, l'absence de cadre juridique, de protection de données personnelles, de tremperie ou d'arnaque de la part du vendeur ou encore de sécurisation technique des réseaux numériques et des transactions.

La partie inférieure gauche du diagramme regroupe les individus qui ne se connectent que peu ou pas du tout et par conséquent n'achètent pas en ligne. Se pose encore une fois la question des fractures numériques de premier et deuxième degré. Le non-achat en ligne pourrait être expliqué soit par un problème d'équipement relatif au non accès aux technologies d'information et de communication soit par un problème de connaissances et d'habiletés cognitives qui peuvent être un réel frein à aux usages productifs du numérique.

Pour les cyber-acheteurs, les raisons qui sous-tendent l'intégration du virtuel dans leurs habitudes de consommation sont liées à « l'utilité perçue » et à « la facilité d'utilisation perçue » (Davis, 1989). En effet, parmi les 34\% des individus qui achètent en ligne, $93 \%$ le justifie par le fait que « l'achat en ligne facilite la comparaison des offres », $85 \%$ déclarent que «L'achat sur Internet représente un gain de temps », etc. Ce résultat est conforme à la littérature qui considère que l'utilité dans le cadre du e-commerce serait la plus-value dégagée par l'expérience de l'achat en ligne. Les travaux de Kim et al. (2003) et Bisdee (2007) sur les intentions comportementales envers l'achat en ligne montrent que les consommateurs auraient des attitudes favorables pour les sites de vente en ligne offrant les services les plus utiles et non disponibles par le biais du shopping traditionnel.

Concernant la facilité d'utilisation perçue, Davis (1989) montre dans ses travaux qu'une technologie qui est perçu comme étant facile à utiliser est plus susceptible d'être acceptée par les utilisateurs tandis qu'une technologie perçue comme complexe sera plus lente à adopter. Dans le contexte marocain, la facilité d'utilisation est un déterminant de la confiance en ligne. Ainsi, la propension d'acheter en ligne sera d'autant plus importante que l'effort consenti pour effectuer la transaction est faible. Dans l'enquête, les résultats à plat montrent que pour $18 \%$ de l'échantillon interrogé des villes de Meknès et de Casablanca, le refus d'achat en ligne serait lié à la complexité de la procédure dans la mesure où ils avancent « ne pas savoir comment faire pour effectuer à un achat en ligne ».

\section{Conclusion}

L'objectif de cet article est de déterminer empiriquement les origines de la confiance/méfiance des consommateurs marocains à l'égard du commerce électronique. 
L'analyse factorielle des correspondances multiples a eu pour objectif l'exploration statistique des données qualitatives obtenues de l'administration d'un questionnaire dans les trois villes. On a cherché à représenter dans un espace de dimension faible, en l'occurrence deux, les nuages de points individus décrits par les variables en utilisant les corrélations existant en elles. Autrement dit, on a étudié les proximités entre individus décrits par les variables ainsi que les proximités entre les modalités de ces variables afin de regrouper les répondants en classes homogènes.

Une analyse factorielle en composantes multiples (ACM) a été réalisée sur l'ensemble des individus et des variables de l'échantillon. Les résultats obtenus viennent appuyer les constats de l'approche descriptive ainsi que des prédictions de départ. On a constaté l'existence d'une méfiance commune envers l'achat en ligne chez les individus localisés dans les trois villes. En effet, les facteurs influençant la perception du risque sont ceux liés à la sécurité des paiements et la fiabilité des vendeurs en ligne. Un autre facteur influençant négativement l'intention d'achat où la poursuite d'achat est le niveau de difficulté perçu, que ça soit pour un achat initial où pour une expérience passée d'achat.

De plus, ces ACM ont permis de classer les individus en trois groupes homogènes selon les variables les plus discriminantes. Les cyberconsommateurs confiants qui achètent sans réserve, les cyber-consommateurs méfiants qui achètent en ligne mais qui pensent être conscients du danger que représente l'achat virtuel et les consommateurs présentant une aversion au commerce électronique.

\section{References :}

1. Allagui, A. \& Temessek A. (2005). La fidélité des utilisateurs des services d'un portail Internet : Proposition et test d'un modèle intégrateur. Actes de l'International Congress of Marketing Trends, Paris.

2. Bart, Y., Venkatesh, S., Fareena, S. \& Urban, G.L. (2005). Are the drivers and role of online trust the same for ail Web sites and consumers? A large-scale exploratory empirical study, Journal of Marketing, 69(4), 133-152.

3. Belanger, F., Hiller, J.S. \& Smith, W.I. (2002). Trustworthiness in electronic commerce: The role of privacy, security, and site attributes. Journal of Strategic Information Systems, 2(3), 245-270.

4. Beneke, J., Scheffer, M-K. \& Du, W. (2010). Beyond Price - An Exploration into the Factors That Drive Young Adults to Purchase Online. International Journal of Marketing Studies, 2(2).

5. Benyoussef, A. (2004). Les Quatre Dimensions De La Fracture Numérique. La Découverte Réseaux, 127/128, 181-209. 
6. Bèzes, C. (2011). Types de risques perçus et réducteurs de risques dans le commerce électronique : le cas du site Fnac.com. Management et avenir, 48(8), 404-422.

7. Bhatnagar, A., Misra, S. \& Rao, R. (2000). On risk, convenience, and Internet shopping behavior. Communications of the ACM, 43(11), 98105.

8. Bisdee, D. (2007). Consumer Attitudes Review, Office of Fair Trading, June, 1-147.

9. Chang, H.H. \& Chen, S.W. (2008). The impact of online store environment cues on purchase intention. Online Information Review, 32(6), 818-841.

10. Chang, Y.S. \& Fang, S.R. (2013). Antecedents and Distinctions between Online Trust and Distrust: Predicting High and Low-Risk Internet Behaviors. Journal of Electronic Commerce Research, 14(2), 149-168.

11. Chellappa, R. K. (2002). Customers' Trust in Electronic Commerce Transactions: The Role of Perceived Privacy and Perceived Security. Atlanta: Emory University.

12. Chouk, L. \& Perrien, J. (2005). La confiance du consommateur vis-àvis d'un marchand Internet: Proposition d'une échelle de mesure. Revue Française de Marketing, 205, 5-20.

13. Corbitt, B.J., Thanasankit, T. \& Yi, H. (2003). Trust and e-commerce: A study of consumer perceptions. Electronic Commerce Research and Applications, 2(3), 203-215.

14. Corritore, C.L., Kracher, B. \& Wiedenbeck, S. (2003). On-line trust: Concepts, evolving themes, a model. International Journal of HumanComputer Studies, 58, 7-37.

15. Davis, F.D. (1989). Perceived usefulness, perceived ease of use, and user acceptance of information technology. MIS Quarterly, 13(3), 31940.

16. Demangeot, C. \& Broderick, A. (2007). Conceptualising consumer behaviour in online shopping environments. International Journal of Retail \& Distribution Management, 35.

17. Deutsch, M. (1962). Cooperation and trust: Some theoretical notes. Nebraska Symposium on Motivation, 10, 275-318.

18. Dillon, A. \& Morris, M. G. (1999). Power, Perception and Performance: From usability Engineering to technology acceptance with the P3 model of user response, $43^{\text {rd }}$ Annual Conference of the Human Factors and Ergonomics Society, Santa Monica, CA: HFES, 1999. 
19. Donada, C. \& Nogatchewsky, G. (2007). La confiance dans les relations interentreprises: Une revue des recherches quantitatives. Revue française de gestion, 175(6), 111-124.

20. Flanagin, A.J. \& Metzger, M.J. (2003). The Perceived Credibility of Personal Webpage Information as Influenced by the Sex of the Source. Computers in Human Behavior,19(6), 683-701.

21. Forsythe, S. \& Shi, B. (2003). Consumer patronage and risk perceptions in Internet shopping", Journal of Business Research, 56, 867-875.

22. Ganguly, R., Dash, S.R., Cyr, D. \& Head, M. (2010). The effects of Website design on purchase intention in online shopping: The mediating role of trust and the moderating role of culture. International Journal of Electronic Business, 8(4/5), 302-330.

23. Gefen, D. (2000). E-Commerce: The role of familiarity and trust, The International Journal of Management Science, 28(6), 725-737.

24. Grazioli, S. \& Jarvenpaa, S. (2000). Perils of Internet fraud: an empirical investigation of deception and trust with experienced Internet consumers. IEEE Transactions on Systems, Man, and Cybernetics, 30(4), 395-410.

25. Guibert, N. (1999). La confiance en marketing: Fondements et applications. Recherche et Applications en Marketing, 144(1), 1-19.

26. Gurviez, P. \& Korchia, M. (2002). Proposition d'une échelle multidimensionnelle de la confiance dans la marque. Recherche et Applications en Marketing, 17(3), 41-58.

27. Halaweh, M. (2012). Modeling User Perceptions Of E-Commerce Security Using Partial Least Square. Journal of Information Technology Management, 23(1).

28. Hargittai, E. (2002). Second-Level Digital Divide: differences in People's Online Skills. First Monday, 7(4).

29. Head, M. \& Hassanein, K. (2002). Trust in e-Commerce: Evaluating the Impact of Third- Party Seals. Quarterly Journal of Electronic Commerce, 3, 307-325.

30. Hess, J. \& Story, L. (2005). Trust-based commitment: Multidimensional consumer-brand relationships. Journal of Consumer Marketing, 22(6), 313-322.

31. Hine, C. \& Eve, J. (1998). Privacy in the marketplace. The Information Society, 14, 253-262.

32. Hu, X., Wu, G. \& Zhang, H. (2010). The effects of Web assurance seals on consumers' initial trust in an online vendor: A functiona1 perspective. Decision Support Systems, vol. 48, 407-418. 
33. Hussin C., A., Macaulay, L. \& Keeling, K. (2006). The Importance Ranking of Trust Attributes in e-Commerce Website, 11th PacificAsia Conference on Information Systems, 2006.

34. Jarvenpaa, S.L., Tractinsky, N. \& Vitale, M. (2000). Consumer trust in an Internet Store: A crosscultural validation. Journal of Computer Mediated Communication, 5(2), 1-35.

35. Kamari, F. \& Kamari, S. (2012). Trust in Electronic Commerce: A New Model for Building Online Trust in B2C. European Journal of Business and Management, 4(10).

36. Kim, DJ., Ferrin, D.L. \& Rao, H.R. (2009). Trust and satisfaction, two stepping stones for successful e-commerce Relationship: A longitudinal exploration, Information Systems Research, 20(2), 237257.

37. Kim, S., Williams, R., \& Lee, Y. (2003). Attitude toward online shopping and retail website quality: a comparison of US and Korean consumers. Journal of International Consumer Marketing. 16(1), 189203.

38. Koehn, D. (2003). The Nature of and Conditions for Online Trust. Journal of Business Ethics, 43, 3-19.

39. Koufaris, M. \& Hampton-Sosa, W. (2004). The development of initial trust in an online company by new customers. Information and Management, 41(3), 377-397.

40. Le Guel, F., Pénard, T. \& Suire, R. (2004). Adoption et usage marchand de l'Internet : une étude économétrique sur données bretonnes. Economie et Prévision, 167, 67-84.

41. Lee, M .K. O. \& Turban, E. (2001). A trust model for consumer Internet shopping. International Journal Of Electronic Commerce, 6(1), 75- 91.

42. Lewis, DJ. \& Weigert, AJ. (1958). Social atomism, holism and trust. The Sociological Quarter/y, 26(4), 455-471.

43. Ltifi, M. \& Gharbi, J-E. (2011). The socio-cognitive theory of trust to electronic trust. Journal of Contemporary Research in Business, 3(7).

44. Marouseau G. (2003). Confiance et commerce électronique : perspectives théoriques et applications pratiques. Actes du $8^{\text {ème }}$ Colloque de l'Association Information et Management (AIM), Grenoble, Mai 2003.

45. Mayer, R. C., Davis, J. H. \& Schoorman, F. C. (1995). An integrative model of organizational trust. Academy of Management Review, 20(3), 709-734.

46. McAllister, D. (1995). Affect and cognition-based trust as foundations for interpersonal cooperation in organization. Academy of Management Journal. 18(1), 24-59. 
47. McKnight, D. H., Choudhury, V. \& Kacmar, C. (2002). Developing and validating trust measures for e-commerce: An integrative typology. Information Systems Research, 13(3), 334-359.

48. McKnight, D.H. \& Chervany, N.L. (2001). What trust means in ecommerce customer relationships: An interdisciplinary conceptual typology. International Journal of Electronic Commerce, 6(2), 35-59.

49. McKnight, H.D., Choudhury, V. \& Kacmar, C. (2002). The Impact of Initial Consumer Trust on Intentions to Transact with a Web Site: A Trust Building Model. Journal of Strategic Information Systems, 11, 297-323.

50. Mitchell, V-W. (1999). Consumer perceived risk: conceptualizations and models. European Journal of Marketing. 33(1/2), 163-195.

51. Miyazaki, A. D. \& Fernandez, A. (2001). Consumer Perception of Privacy and Security Risks for Online Shopping. The Journal of Consumer Affairs, 35(1), 27-44.

52. Moore, R., Stammerjohan, C. \& Coulter, R. (2005). Banner advertiserweb site context congruity and colour effects on attention and attitudes. Journal of Advertising, 34(2), 71-84.

53. Nguyen, N. \& Leblanc, G. (2001). Corporate image and corporate reputation in consumers' retention decision in services. Journal of retailing and Consumer Services, 8(3), 227-236.

54. Nissenbaum, H. (2001). Securing Trust Online: Wisdom or Oxymoron?. Boston University Law Review, 81(3), 635-664.

55. Novak, T.P. \& Peralta, M. (2000). Building consumer trust online. Communications of the Association for Computing Machinery, 43(4), 100-122.

56. Oukarfi, S. (2013). L'usage de l'internet au Maroc: essai de mesure de la fracture numérique de deuxième degré. International Journal of Innovation and Applied Studies, 2(2), 118-130.

57. Papadopoulou, P. \& Martakos, D. (2008). Trust in e-commerce: Conceptualization and operationalization issues. IADIS International Conference e-Commerce.

58. Pavlou, P.A. (2003). Consumer Acceptance of Electronic Commerce: Integrating Trust and Risk with the Technology Acceptance Model. International Journal of Electronic Commerce, 7(3), 101-134.

59. Rajaobelina, L., Boyer, K., Ricard, L. \& Toufaily, E. (2011). Les communautés virtuelles dans le secteur touristique sont-elles au service du marketing relationnel?. Actes du Congrès de l'Association des Sciences Administratives du Canada, Montréal, Québec, 2-5 juillet 2011. 
60. Rousseau, D. M., Burt, R. S., \& Camerer, C. (1998). Not so different after all: a cross-discipline view of trust. Journal of Academy Management Review, 23(3), 393-404.

61. Singh, V. \& Sabol, B. (2002). Consumer trust, value, and loyalty in relational exchanges. Journal of Marketing, 66(1), 15-37.

62. Singh, V. (2004). Facteurs d'utilisation d'Internet à la maison. Bulletin d'analyse - Région rurales et petites villes du Canada. 5(1).

63. Stremtan, F., \& Muntean, A. (2008). Measuring quality of the services provided by the conunercial Web sites. Annales Universitatis Apulensis Series Oeconomica, 2(10), 53-56.

64. Suh, B. \& Han I. (2003). The Impact of Consumer Trust and Perception of Security Control on the Acceptance of Electronic Commerce. International Journal of Electronic Commerce, 7(3), 135161.

65. Van Dijk J. (2005). The Deepening Divide Inequality in the Information Society. Sage Publications, Thousand, Oaks CA, London/New Delhi. Doi: 10.4135/9781452229812.

66. Wang, W. \& Benbasat, L. (2005). Integrating TAM with trust to explain online recommendation agent adoption. Journal of Association for Information Systems, 6(3), 72-101.

67. Wang, Y.D. \& Emurian, H.H. (2004). An overview of online trust: Concepts, elements and implications. Computers in Human Behavior, 21(1), 105-125.

68. Warschauer, M. (2003). Technology and social inclusion. Rethinking the digital divide, MIT Press, Massachusetts.

69. Yoh, E., Damhorst, M. L., Sapp, S., \& Laczniak, R. (2003). Consumer adoption of the Internet: the case of apparel shopping. Psychology and Marketing, 20(12), 1095-1118. 